\title{
EVALUACIÓN DE ECUACIONES QUE UTILIZAN CISTATINA C PARA ESTIMAR FILTRADO GLOMERULAR EN ADULTOS SANOS CANDIDATOS A DONANTES RENALES
}

\author{
EVALUATION OF EQUATIONS USING CYSTATIN C FOR ESTIMATION OF THE GLOMERULAR FILTRATION \\ RATE IN HEALTHY ADULT POPULATION OF CANIDATES FOR KIDNEY DONORS
}

\section{Gisel I. Medina Arnaudo ${ }^{1}$, Pablo R. Luján ${ }^{1}$, Carlos Chiurchiu ${ }^{2}$, Jorge De la Fuente ${ }^{2}$, Raúl H. Capra ${ }^{1}$}

\begin{abstract}
Resumen:
La valoración del Índice de Filtrado Glomerular (IFG) es fundamental para la selección de potenciales donantes renales. Para su medición existen métodos poco prácticos y complejos, por lo cual se han desarrollado ecuaciones de estimación del IFG. Objetivo: Evaluar el rendimiento diagnóstico de ecuaciones de estimación del IFG a partir de Creatinina y Cistatina $C$ en una población de adultos sanos candidatos a donantes renales. Materiales y métodos: Se llevó a cabo un estudio transversal en 72 pacientes de ambos sexos de entre 18 y 75 años de edad, candidatos a donantes renales. Se les midió el IFG a través del clearance de lotalamato no marcado (IFGm). Se evaluaron cuatro ecuaciones de estimación del FG (IFGe): MDRD, del estudio "Modification of Diet in Renal Disease"; CKD-EPI-Creatinina (CKD-EPI-CREAT) y CKD-EPI-Creatinina y Cistatina C (CKD-EPICREAT+CIS), desarrolladas por el grupo "Chronic Kidney Disease Epidemiology Collaboration"; y la que incluye Creatinina y Cistatina C propuesta por Stevens y col. (STEVENS-CREAT+CIS). Se analizó el desempeño de cada ecuación con respecto al IFGm. Resultados: Las ecuaciones CKD-EPI-CREAT+CIS, STEVENS-CREAT+CIS y CKD-EPI-CREAT mostraron ser más exactas (bias $=1,93 ; \quad-0,71 ; \quad-1,53 \quad \mathrm{~mL} / \mathrm{min} / 1,73 \mathrm{~m} 2$ respectivamente) que MDRD (bias $=-10,36$ $\mathrm{mL} / \mathrm{min} / 1,73 \mathrm{~m} 2$ ). El mejor rendimiento diagnóstico lo mostró el IFGe CKD-EPI-CREAT+CIS $(L R(+)=9,8$; "Positive Likelihood Ratios"), seguido por IFGe STEVENS-CREAT+CIS $(\operatorname{LR}(+)=4,7)$ y luego por IFGe CKD-EPI-CREAT $(\operatorname{LR}(+)=3,7)$. Discusión: Las ecuaciones CKD-EPI-CREAT+CIS, STEVENS-CREAT+CIS y CKD-EPI-CREAT demostraron tener un desempeño claramente superior a MDRD para estimar el IFG en la población estudiada.
\end{abstract}

Palabras clave: cistatina C; estimación del Índice de filtrado Glomerular; donantes renales.

\begin{abstract}
:
The determination of the glomerular filtration rate (GFR) is critical for the selection of potential kidney donors. Methods of measurement of GFR are impractical and complex, which led to development of equations to estimate GFR. Objective: To evaluate of the performance of Creatinine and Cystatin $C$ based equation for estimation of GFR in healthy adult population of candidates for kidney donors. Materials and Methods: a cross-sectional study was performed in 72 adult patients evaluated as candidates for kidney donors. GFR was measured by nonradiolabeled lothalamate clearence (mGFR). GFR was estimated by the Modification of Diet in Renal Disease (eGFR MDRD) equation, Chronic Kidney Disease Epidemiology Collaboration study (eGFR CKD-EPICREAT, eGFR CKD-EPI-CREAT+CYSC) and the equation proposed by Stevens et al. (STEVENSCREAT+CYSC). The overall performance of all equations was analyzed. Results: eGFR CKD-EPICREAT+CYSC, eGFR STEVENS CREAT+CYSC and eGFR CKD-EPI-CREAT presented lower bias (1,93; 0,$71 ;-1,53 \mathrm{~mL} / \mathrm{min} / 1,73 \mathrm{~m} 2$ respectively) compared with eGFR MDRD (bias $=-10,36 \mathrm{~mL} / \mathrm{min} / 1.73 \mathrm{~m} 2)$. This study demonstrated that the best diagnostic performance was shown by eGFR CKD-EPICREAT+CYSC $(\operatorname{LR}(+)=9,8$; "Positive Likelihood Ratios"), followed by eGFR STEVENSCREAT+CYSC $(\mathrm{LR}(+)=4,7)$ and eGFR CKD-EPICREAT $(\operatorname{LR}(+)=3,7)$. Discussion: CKD-EPICREAT+CYSC, STEVENS-CREAT+CYSC and CKDEPI-CREAT equations demonstrated a clearly higher performance to estimate GFR compared with MDRD in the study population.
\end{abstract}

Key words: cystatin C; estimation of Glomerular Filtration Rate; kidney donors.

1.-Laboratorio de Química Clínica, Hospital Privado Centro Médico de Córdoba, Córdoba, Argentina. E-mail: gisel.medina@hospitalprivadosa.com.ar

2.-Servicio de Nefrología, Programa de Transplante Renal, Hospital Privado Centro Médico de Córdoba, Córdoba, Argentina.. 


\section{INTRODUCCIÓN}

La determinación del filtrado glomerular (FG) es considerada el mejor índice para evaluar la función renal tanto en individuos sanos como enfermos. Más aún, su valoración es fundamental para identificar y estratificar pacientes con enfermedad renal crónica (ERC), ajustar dosis de drogas nefrotóxicas y seleccionar potenciales candidatos a ser donantes renales ${ }^{(1,2)}$.

Los valores del Índice de Filtrado Glomerular (IFG) varían de acuerdo a la edad, sexo y superficie corporal; siendo aproximadamente de $130 \mathrm{~mL} / \mathrm{min}$ por $1,73 \mathrm{~m}^{2}$ y $120 \mathrm{~mL} / \mathrm{min}$ por $1,73 \mathrm{~m}^{2}$ en hombres y mujeres adultos sanos respectivamente ${ }^{(3,4)}$.

Para valorar el IFG con un alto grado de exactitud es necesario el uso de técnicas invasivas basadas en la medición del clearance de marcadores exógenos; como el clearance de Inulina que ha sido considerado el método patrón; o el de marcadores isotópicos o no isotópicos, que en la actualidad son utilizados como métodos de referencia. Sin embargo, estas técnicas tienen un uso limitado en la práctica clínica habitual, ya que son métodos laboriosos y costosos que implican un consumo de tiempo elevado y pueden resultar incómodos para el paciente; su aplicación queda relegada a situaciones puntuales: pacientes con masa muscular alterada (amputados, parálisis), índice de masa corporal (IMC) extremo, posibles donantes renales, dosificación de fármacos nefrotóxicos y en investigación ${ }^{(5,6)}$.

En la práctica clínica, la Creatinina es el marcador endógeno más utilizado para evaluar el FG, ya que su medición es rápida y de bajo costo. Sin embargo, se debe tener en cuenta que la concentración plasmática de Creatinina depende de la edad, sexo, raza, estado nutricional y masa muscular de cada individuo; tiene una sensibilidad diagnóstica insuficiente para detectar cambios leves a moderados de la función renal; errores en la recolección de orina de 24 hs llevan a un clearance de Creatinina inexacto y la secreción tubular de Creatinina da como resultado una sobreestimación del IFG de aproximadamente un $20 \%$ cuando éste tiene valores normales y esta brecha se agranda en presencia de insuficiencia renal. Estos factores pueden conducir a una incorrecta interpretación del FG ${ }^{(6-8)}$. Por esto, y con el afán de contar con una simple herramienta para evaluar el $F G$, se han desarrollado ecuaciones matemáticas que estiman el IFG a partir del valor de Creatinina plasmática, variables demográficas y antropométricas. En la actualidad las fórmulas surgidas del estudio Modification of Diet in Renal Disease (MDRD) y la desarrollada por el grupo de CKD Epidemiology Collaboration (CKDEPI) son las más usadas ${ }^{(9,10)}$.

Estas fórmulas fueron validadas en poblaciones de pacientes con características demográficas y raciales específicas, por lo tanto el uso de las mismas en otras poblaciones requiere de una validación previa.

Las limitaciones del uso de la Creatinina para evaluar el FG han llevado a la búsqueda de nuevos marcadores endógenos. Con esta visión, en los últimos tiempos ha sido objeto de estudio de muchas publicaciones lo que ya en el año 1985 plantearon Simonsen y col.: la concentración sérica de Cistatina $\mathrm{C}$ como medida del IFG ${ }^{(11)}$. La Cistatina $\mathrm{C}$ es una proteína básica no glicosilada de bajo peso molecular que pertenece a la superfamilia de inhibidores de cisteína - proteasas, sintetizada por todas las células nucleadas del organismo. A pH fisiológico tiene carga positiva que permite que filtre libremente por el glomérulo, para luego ser reabsorbida y catabolizada por las células tubulares, por lo que no retorna al torrente sanguíneo. Su producción es constante y no dependería de la edad, sexo, masa muscular o dieta ${ }^{(12-14)}$. Estas características hacen que sea posible considerarla como un marcador endógeno alternativo y prometedor para evaluar la función renal. Más aún, varios estudios sugieren que la concentración sérica de Cistatina $C$ sería un indicador con mayor sensibilidad que la concentración sérica de Creatinina para detectar pequeños cambios en la función renal ${ }^{(15,16)}$. Sin embargo, surge la necesidad de estimar el IFG a partir de Cistatina $C$ y no simplemente analizar una concentración en suero ${ }^{(17)}$. En este sentido, varios autores han desarrollado ecuaciones matemáticas validadas en diferentes poblaciones. Stevens y col. han publicado dos fórmulas de estimación, una que incluye como variables a la Cistatina $C$, edad, sexo y raza, y en la otra además a la Creatinina ${ }^{(18)}$. Hoek y col. y Larsson y col. han propuesto ecuaciones que sólo contemplan el valor de Cistatina $C$ $(15,19)$. En el año 2012 el grupo de CKD Epidemiology Collaboration (CKD-EPI) ha propuesto dos fórmulas de estimación, en una incluye valores de Cistatina $C$, edad y sexo; en la segunda tiene en cuenta además la concentración de Creatinina plasmática y la raza ${ }^{(20)}$.

Con esta gran oferta de fórmulas disponibles, muchos autores han estudiado el desempeño de algunas de ellas en poblaciones específicas de pacientes, concluyendo que la inclusión de 
Cistatina $C$ en las fórmulas les otorga FG estimados con mayor sensibilidad y especificidad que los calculados sólo con Creatinina ${ }^{(21-25)}$

El objetivo del presente trabajo es evaluar el rendimiento diagnóstico de ecuaciones de estimación del IFG a partir de Creatinina y Cistatina $C$ en una población de adultos sanos candidatos a donantes renales.

\section{MATERIALES Y MÉTODOS}

Se llevó a cabo un estudio transversal en 72 pacientes de ambos sexos de entre 18 y 75 años de edad, candidatos a donantes renales que participaron del Programa de Transplante Renal del Hospital Privado Centro Médico de Córdoba.

El presente trabajo cuenta con la aprobación del Comité de Ética del Hospital Privado Centro Médico de Córdoba.

Se tuvieron en cuenta los siguientes criterios de exclusión:

*presencia de disfunción tiroidea

*concentraciones de Proteína C Reactiva > 0,6 mg/dL

*tabaquismo

*uso de corticoesteroides

*Índice de Masa Corporal (IMC) $<18 \mathrm{~kg} / \mathrm{m}^{2}$ y $>30 \mathrm{~kg} / \mathrm{m}^{2}$

Se registraron y analizaron: edad, sexo, IFG medido a través del clearance de lotalamato no marcado, concentración de Creatinina plasmática $\left(\right.$ Crea $\left._{p}\right)$ y concentración de Cistatina C plasmática $\left(\mathrm{Cis}_{\mathrm{p}}\right)$.

Para medir el IFG (IFGm) se administró una solución de lotalamato previamente preparada a partir de una concentración de $282 \mathrm{mg} / \mathrm{dL}$ del mismo; en los tiempos correspondientes se tomaron muestras de plasma con heparina como agente anticoagulante y se recolectaron muestras de orina en recipientes estériles. El lotalamato en plasma y orina fue medido por cromatografía líquida de alta resolución (HPLC, del inglés "High Performance Liquid Chromatography") utilizando un cromatógrafo Gilson (Middleton, WI) con un detector de rayos UV/Visible modelo 189 y una columna Phenomenex (20 mm - ID $4.6 \mu \mathrm{m}$, Torrance, CA) C18. Los resultados del clearance de lotalamato se expresaron en $\mathrm{mL} / \mathrm{min} / 1,73 \mathrm{~m}^{2}$.

Las determinaciones de $\mathrm{Crea}_{\mathrm{p}}$ y $\mathrm{Cis}_{\mathrm{p}}$ se llevaron a cabo en las muestras basales de plasma en un autoanalizador Modular P (Roche Diagnostics). Para la Crea $_{p}$ se utilizó el método cinético de Jaffé, trazable con el método de referencia: Espectrometría de Masa con Dilución Isotópica (IDMS, del inglés "Isotope Dilution Mass Spectrometry"); los resultados se expresaron en $\mathrm{mg} / \mathrm{dL}$. La Cis fue determinada con un ensayo inmunoturbidimétrico potenciado con partículas de látex recubiertas con anticuerpos anti-Cistatina, trazable frente al material de referencia ERM-DA471/IFCC; los resultados se expresaron en $\mathrm{mg} / \mathrm{L}$.

Para la estimación del IFG (IFGe) se utilizaron las ecuaciones que se especifican en la Tabla 1. Los valores de IFGe se expresaron en $\mathrm{mL} / \mathrm{min} / 1,73 \mathrm{~m}^{2}$.

TABLA 1- Ecuaciones de estimación del IFG.

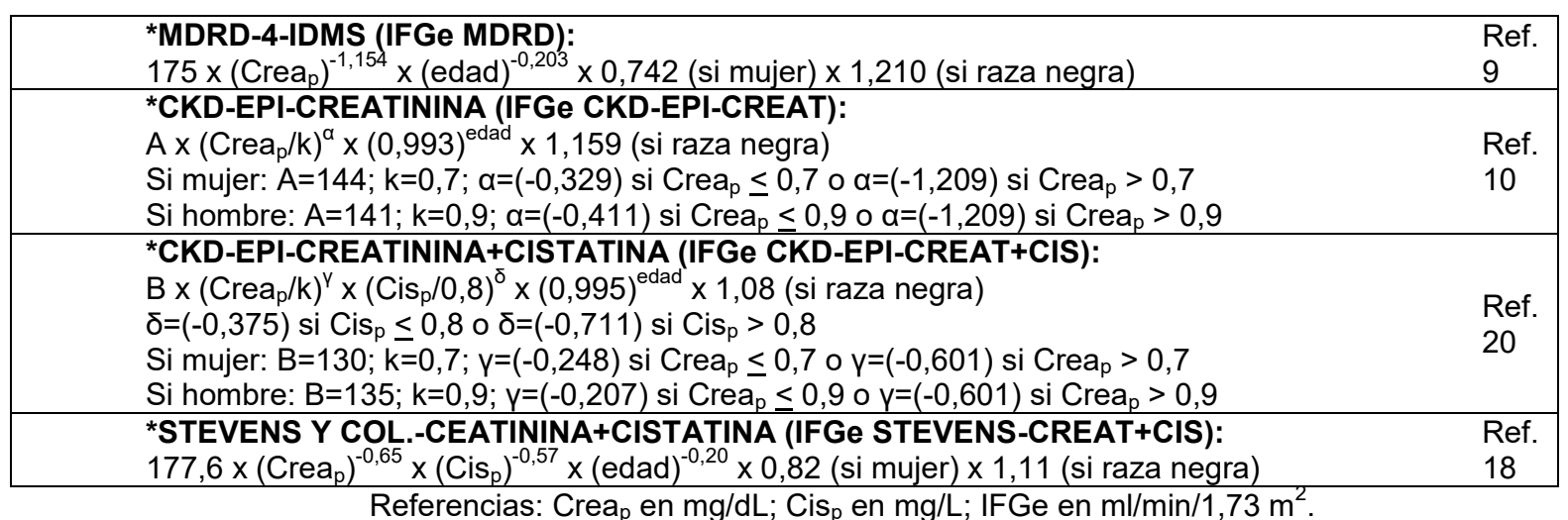

El análisis estadístico de los datos se llevó a cabo utilizando los programas InfoStat y MedCalc. Se calcularon las medidas descriptivas de la población y de los IFG (IFGm e IFGe). Se consideró 
como método de referencia al clearance de lotalamato (IFGm), con respecto al cual se obtuvieron bias (diferencias entre IFGm e IFGe), precisión (Coeficientes de Variación Porcentual, CV\%), curvas de regresión y coeficientes de correlación de Pearson para cada IFGe. Para evaluar el grado de concordancia entre los valores estimados y los medidos se realizaron los gráficos que Bland y Altman describen (26). Se construyeron curvas ROC (del inglés "Receiver Operating Characteristic", Característica Operativa del Receptor) a partir de las cuales se obtuvieron diferentes parámetros diagnósticos de cada ecuación de estimación para la detección de un IFGm menor a $90 \mathrm{~mL} / \mathrm{min} / 1,73 \mathrm{~m}^{2}$.

Valores de probabilidades menores a 0,05 fueron considerados significativos $(p<0,05)$.

\section{RESULTADOS}

Las características de la población estudiada se detallan en la Tabla 2. Los valores de FG, tanto medidos como estimados, arrojaron una distribución normal según el test de normalidad D'Agostino-Pearson ( $p>0,05)$.

El IFGe (MDRD) presentó el mayor bias $\left(-10,36 \mathrm{~mL} / \mathrm{min} / 1,73 \mathrm{~m}^{2}\right)$ con respecto al IFGm, demostrando una subestimación del FG estadísticamente significativa $(p<0,05)$; y la precisión de esta ecuación fue la menor $(C V \%=16,59)$. Los valores de $F G$ estimados por el resto de las ecuaciones evaluadas (IFGe CKD-EPI-CREAT, IFGe CKD-EPI-CREAT+CIS e IFGe STEVENSCREAT+CIS) no presentaron diferencias estadísticamente significativas con respecto a los valores de FG medidos $(p>0,05)$. El IFGe (STEVENS-CREAT+CIS) fue el de menor bias $(-0,71$ $\left.\mathrm{ml} / \mathrm{min} / 1,73 \mathrm{~m}^{2}\right)$. El IFGe (CKD-EPI-CREAT) fue el de mayor precisión en sus datos $(\mathrm{CV} \%=14,70)$. Tabla 3.

TABLA 2- Características de la población estudiada.

\begin{tabular}{|cc|}
\hline Variable & Valores \\
\hline Número $(\mathrm{n})$ & 72 \\
\hline \multirow{2}{*}{ Edad (años), media $\pm \mathrm{DE}$} & $45,01 \pm$ \\
& 13,40 \\
\hline Sexo femenino $(\mathrm{n})$ & 40 \\
\hline \multirow{2}{*}{ Crea $_{\mathrm{p}}(\mathrm{mg} / \mathrm{dL})$, media $\pm \mathrm{DE}$} & $0,85 \pm$ \\
& 0,19 \\
\hline \multirow{2}{*}{$\mathrm{Cis}_{\mathrm{p}}(\mathrm{mg} / \mathrm{L})$, media $\pm \mathrm{DE}$} & $0,83 \pm$ \\
& 0,16 \\
\hline $\mathrm{IFGm}$ lotalamato $\left(\mathrm{mL} / \mathrm{min} / 1,73 \mathrm{~m}^{2}\right)$, media \pm & $98,52 \pm$ \\
$\mathrm{DE}$ & 14,04 \\
\hline $\mathrm{IFGm}<90 \mathrm{ml} / \mathrm{min} / 1,73 \mathrm{~m}^{2}(\mathrm{n})$ & 23 \\
\hline $\mathrm{IFGm} \geq 90 \mathrm{ml} / \mathrm{min} / 1,73 \mathrm{~m}^{2}(\mathrm{n})$ & 49 \\
\hline
\end{tabular}

TABLA 3- Rendimiento general de las ecuaciones estudiadas.

\begin{tabular}{|c|c|c|c|c|}
\hline Variable & $\begin{array}{l}\text { IFGe } \\
\text { MDRD }\end{array}$ & $\begin{array}{l}\text { IFGe } \\
\text { CKD-EPI- } \\
\text { CREAT }\end{array}$ & $\begin{array}{l}\text { IFGe } \\
\text { CKD-EPI- } \\
\text { CREAT+CIS }\end{array}$ & $\begin{array}{l}\text { IFGe } \\
\text { STEVENS } \\
\text { CREAT+CIS }\end{array}$ \\
\hline $\begin{array}{l}\text { Media } \\
\mathrm{mL} / \mathrm{min} / 1,73 \mathrm{~m}^{2}\end{array}$ & 86,50 & 94,09 & 97,71 & 94,17 \\
\hline $\begin{array}{l}\mathrm{DE} \\
\mathrm{mL} / \mathrm{min} / 1,73 \mathrm{~m}^{2}\end{array}$ & 14,35 & 13,83 & 14,98 & 15,13 \\
\hline IC $95 \%$ & $\begin{array}{l}{[58,37 ;} \\
114,63]\end{array}$ & $\begin{array}{l}{[66,98 ;} \\
121,20]\end{array}$ & $\begin{array}{l}{[68,35 ;} \\
127,07]\end{array}$ & $\begin{array}{l}{[64,52 ;} \\
123,82]\end{array}$ \\
\hline $\begin{array}{l}\text { Bias } \\
\left(\mathrm{mL} / \mathrm{min} / 1,73 \mathrm{~m}^{2}\right)\end{array}$ & $-10,36$ & $-1,53$ & 1,93 & $-0,71$ \\
\hline $\begin{array}{l}\text { IC } 95 \% \\
\left(\mathrm{~mL} / \mathrm{min} / 1,73 \mathrm{~m}^{2}\right)\end{array}$ & $\begin{array}{l}{[-} \\
18,08 ;- \\
2,65]\end{array}$ & $\begin{array}{l}{[-} \\
4,63 ; 1,58]\end{array}$ & {$[-1,55 ; 5,41]$} & {$[-2,84 ; 1,42]$} \\
\hline
\end{tabular}


Las curvas de regresión y los coeficientes de correlación de Pearson ( $r$ ) de cada IFGe con respecto al IFGm se detallan en la Figura 1. El IFGe (CKD-EPI-CREAT+CIS) arrojó el mayo valor de $r(0,77)$, mientras que el IFGe (STEVENS-CREAT+CIS) el menor $(0,68)$. Sin embargo, se debe tener en cuenta que este coeficiente de correlación evidencia si existe una asociación lineal, con baja sensibilidad para detectar diferencias sistemáticas entre las ecuaciones y el clearance de lotalamato. Todos los IFGe presentaron un $r$ que demostró una asociación lineal estadística con el IFGm $(p<0,001)$.
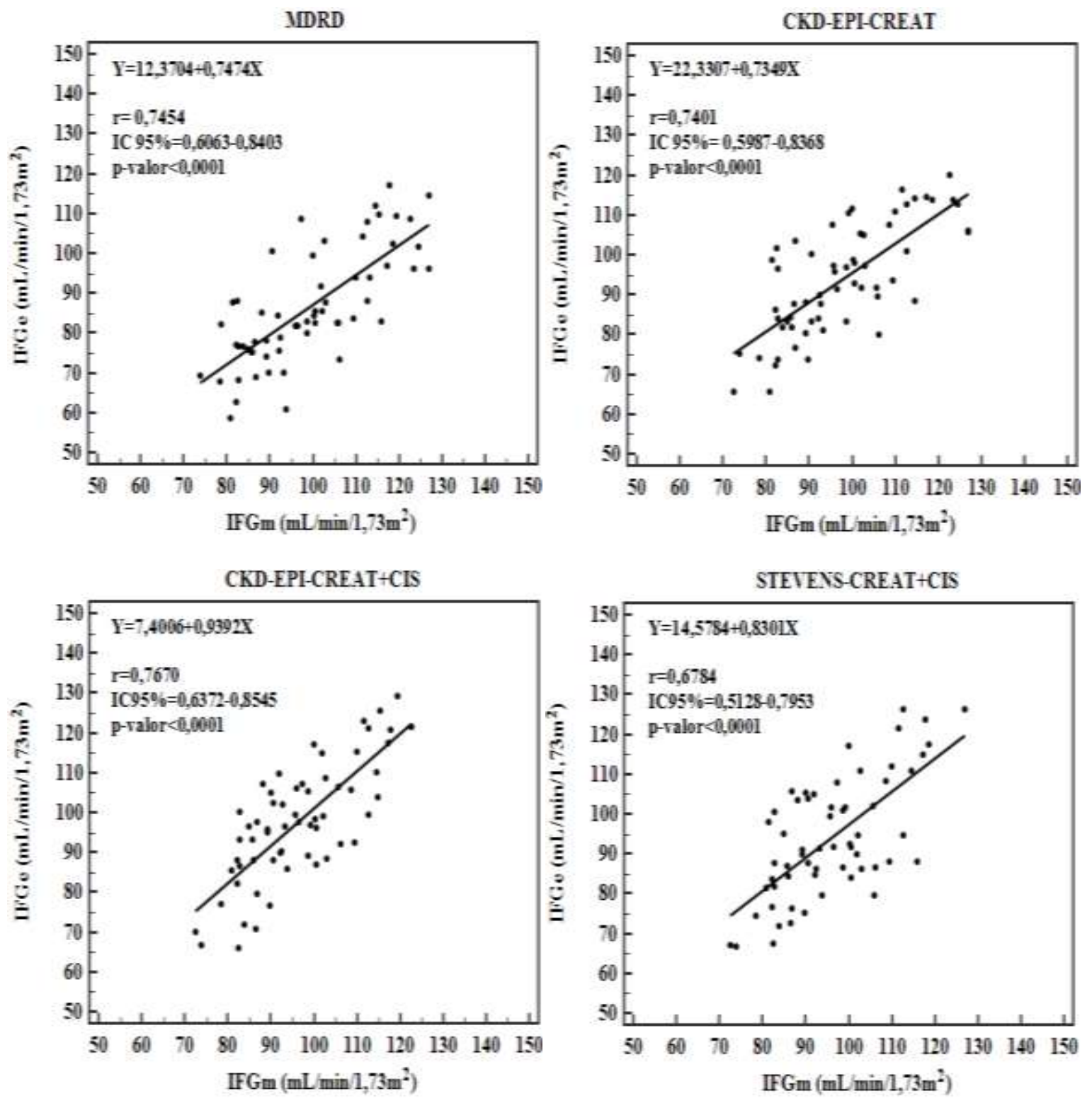

El análisis de Bland-Altman se muestra en la Figura 2. Se puede observar que los IFGe (CKD-EPICREAT+CIS) y (STEVENS-CREAT+CIS) son los que presentaron mayor grado de concordancia con el IFGm; sin embargo el IFGe (CKD-EPI-CREAT+CIS) presentó menor dispersión de los datos que IFGe (STEVENS-CREAT+CIS). 


\section{Cistatina c y filtrado glomerular}

FIGURA 2. Gráficos de Bland Altman para cada IFGe con respecto al IFGm. Se expresa la media de las diferencias entre el IFGe y el IFGm (bias) y los límites de concordancia con un $95 \%$ de confianza.
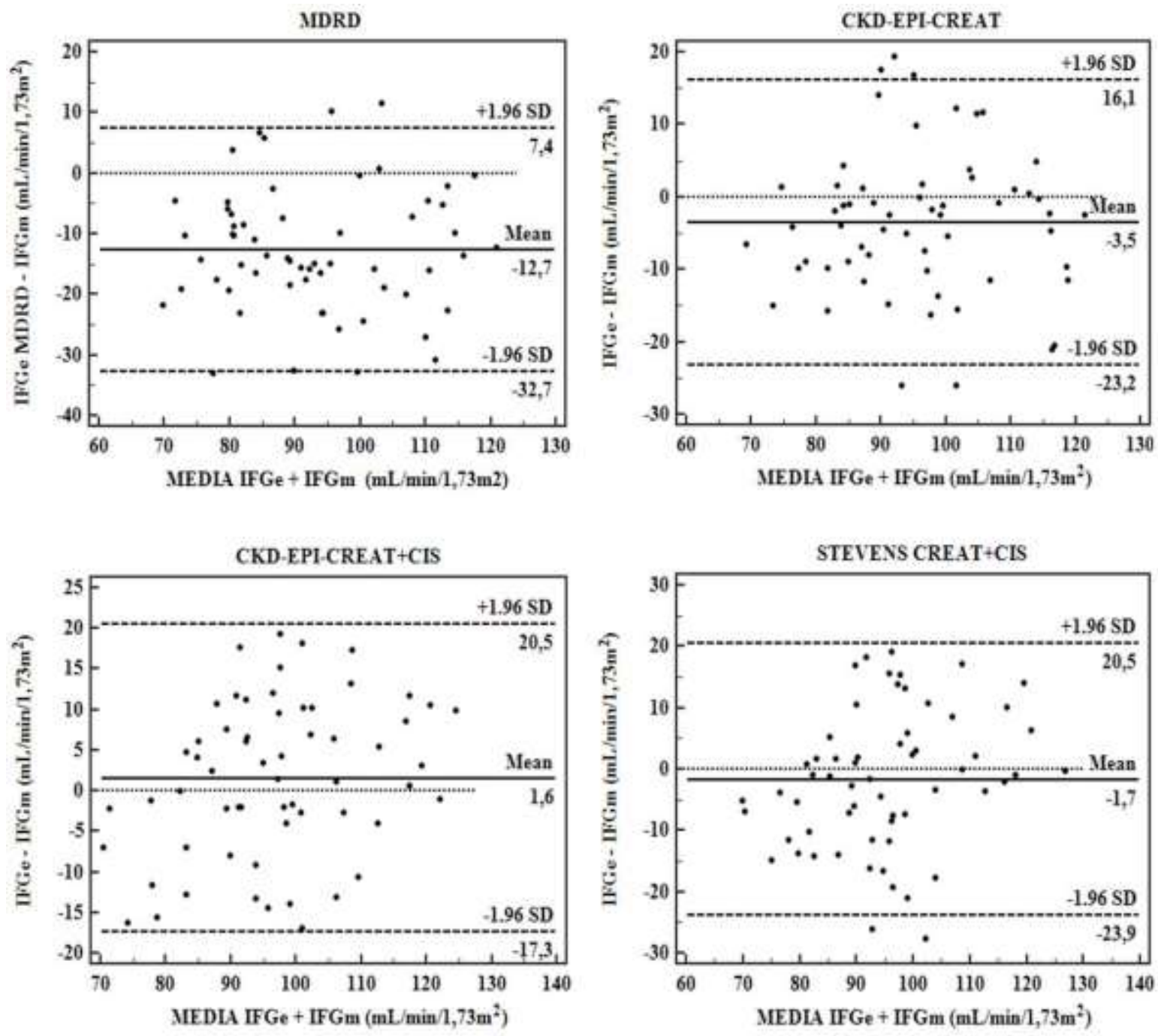

Se utilizaron curvas ROC para evaluar el rendimiento diagnóstico de las diferentes ecuaciones para predecir y categorizar correctamente un IFGm menor a $90 \mathrm{~mL} / \mathrm{min} / 1,73 \mathrm{~m}^{2}$, valor de corte establecido para la selección de candidatos a ser donantes renales. El mejor rendimiento diagnóstico lo mostraron las fórmulas que incorporan Cistatina C; en primer lugar IFGe CKD-EPICREAT+CIS con un $L R(+)=9,8$ (del inglés "Positive Likelihood Ratio", Razón de Verosimilitud Positiva), seguido por IFGe STEVENS-CREAT+CIS con un $L R(+)=4,7$. Sin embargo el IFGe CKDEPI-CREAT evidenció un desempeño muy razonable con un $L R(+)=3,7$. Tabla 4. 
TABLA 4- Rendimiento de las ecuaciones para un IFGm $<90 \mathrm{~mL} / \mathrm{min} / 1,73 \mathrm{~m} 2$

\begin{tabular}{|c|c|c|c|c|}
\hline Variable & $\begin{array}{l}\text { IFGe } \\
\text { MDRD }\end{array}$ & $\begin{array}{l}\text { IFGe } \\
\text { CKD- } \\
\text { EPI- } \\
\text { CREA } \\
\text { T } \\
\end{array}$ & $\begin{array}{l}\text { IFGe } \\
\text { CKD-EPI } \\
\text { CREAT+ } \\
\text { CIS }\end{array}$ & $\begin{array}{l}\text { IFGe } \\
\text { STEVE } \\
\text { NS } \\
\text { CREAT } \\
+ \text { CIS } \\
\end{array}$ \\
\hline $\begin{array}{l}\text { SENSIBILIDA } \\
\mathrm{D}, \% \\
\text { IC } 95 \%\end{array}$ & $\begin{array}{l}90,9 \\
(70,8- \\
98,9)\end{array}$ & $\begin{array}{l}68,2 \\
(45,1- \\
86,1)\end{array}$ & $\begin{array}{c}71,4 \\
(47,8- \\
88,7)\end{array}$ & $\begin{array}{l}72,7 \\
(49,8- \\
89,3)\end{array}$ \\
\hline $\begin{array}{l}\text { ESPECIFICID } \\
\text { AD, } \% \\
\text { IC } 95 \% \\
\end{array}$ & $\begin{array}{l}54,2 \\
(39,2- \\
68,6) \\
\end{array}$ & $\begin{array}{l}81,6 \\
(68,0- \\
91,2) \\
\end{array}$ & $\begin{array}{l}92,7 \\
(80,1- \\
98,5)\end{array}$ & $\begin{array}{l}84,6 \\
(69,5- \\
94,1)\end{array}$ \\
\hline $\begin{array}{l}\text { VPP, } \% \\
\text { IC 95\% }\end{array}$ & $\begin{array}{l}47,6 \\
(32,0- \\
63,6) \\
\end{array}$ & $\begin{array}{l}62,5 \\
(40,6- \\
81,2)\end{array}$ & $\begin{array}{l}83,3 \\
(58,6- \\
96,4)\end{array}$ & $\begin{array}{l}72,7 \\
(49,8- \\
89,3)\end{array}$ \\
\hline $\begin{array}{l}\text { VPN, } \% \\
\text { IC } 95 \%\end{array}$ & $\begin{array}{l}92,9 \\
(76,5- \\
99,1)\end{array}$ & $\begin{array}{l}85,1 \\
(71,7- \\
93,8)\end{array}$ & $\begin{array}{l}86,4 \\
(72,6- \\
94,8)\end{array}$ & $\begin{array}{l}84,6 \\
(69,5- \\
94,1)\end{array}$ \\
\hline $\begin{array}{l}\text { LR(+) } \\
\text { IC 95\% }\end{array}$ & $\begin{array}{l}2,0 \\
(1,4- \\
2,8)\end{array}$ & $\begin{array}{l}3,7 \\
(1,9- \\
7,2)\end{array}$ & $\begin{array}{l}9,8 \\
(3,2- \\
30,0)\end{array}$ & $\begin{array}{l}4,7 \\
(2,2- \\
10,3)\end{array}$ \\
\hline $\begin{array}{l}\text { LR(-) } \\
\text { IC 95\% }\end{array}$ & $\begin{array}{l}0,2 \\
(0,0- \\
0,6)\end{array}$ & $\begin{array}{l}0,4 \\
(0,2- \\
0,7)\end{array}$ & $\begin{array}{l}0,3 \\
(0,2-0,6)\end{array}$ & $\begin{array}{l}0,3 \\
(0,2- \\
0,6)\end{array}$ \\
\hline $\begin{array}{l}\text { CURVAS } \\
\text { ROC, ABC }\end{array}$ & 0,82 & 0,87 & 0,91 & 0,90 \\
\hline $\begin{array}{l}\text { CURVAS } \\
\text { ROC, EE }\end{array}$ & 0,06 & 0,05 & 0,04 & 0,04 \\
\hline $\begin{array}{l}\text { CURVAS } \\
\text { ROC, IC 95\% }\end{array}$ & $\begin{array}{l}0,69- \\
0,91\end{array}$ & $\begin{array}{l}0,75- \\
0,94\end{array}$ & $0,80-0,97$ & $\begin{array}{l}0,79- \\
0,96\end{array}$ \\
\hline
\end{tabular}

Referencias: VPP= valor predictivo positivo; VPN= valor predictivo negativo; IC 95\%= intervalo de confianza del 95\%; LR= del inglés "Likelihood Ratios", Razón de Verosimilitud; ROC= del inglés,"Receiver Operating Characteristic", Característica Operativa del Receptor; $A B C=$ área bajo la curva; EE=error estándar.

\section{DISCUSIÓN}

En el presente trabajo, las ecuaciones CKD-EPI-CREAT+CIS, STEVENS-CREAT+CIS y CKD-EPICREAT, demostraron tener un desempeño claramente superior a MDRD para estimar el IFG en la población estudiada, expresado a través de un menor bias $\left(1,93 ;-0,71 ;-1,53 \mathrm{~mL} / \mathrm{min} / 1,73 \mathrm{~m}^{2}\right.$ respectivamente) con respecto al de la ecuación MDRD $\left(-10,36 \mathrm{~mL} / \mathrm{min} / 1,73 \mathrm{~m}^{2}\right)$ y mejor rendimiento diagnóstico para un IFGm $<90 \mathrm{~mL} / \mathrm{min} / 1,73 \mathrm{~m}^{2}$ evidenciado por las curvas ROC; donde la ecuación CKD-EPI-CREAT+CIS arrojó el valor más alto de $L R(+)=9,8$ y un valor de $L R(-)=0,3$ en la población estudiada, debido a la mayor especificidad de la ecuación en la detección de FG menores a $90 \mathrm{~mL} / \mathrm{min} / 1,73 \mathrm{~m}^{2}$.

Las limitaciones ya conocidas que presenta la ecuación MDRD cuando es utilizada en una población de pacientes sin ERC se evidenciaron en los resultados obtenidos. Presentó la menor exactitud subestimando de manera sistemática los IFGm (bias = -10,36; IC 95\% = -18,08; -2,65). A pesar de que el área bajo su curva $\mathrm{ROC}$ para un $\mathrm{IFGm}<90 \mathrm{~mL} / \mathrm{min} / 1,73 \mathrm{~m}^{2}$ arrojó un valor considerable $(0,82)$ por su alta sensibilidad, su baja especificidad diagnóstica es la que puede provocar una incorrecta evaluación de los IFG en los candidatos a donación renal. Por estas limitaciones, no sería apropiado el uso de la ecuación MDRD para estimar los FG en la población estudiada.

En el presente trabajo, las ecuaciones CKD-EPI-CREAT, CKD-EPI-CREAT+CIS y STEVENSCREAT+CIS mostraron un buen desempeño en la población estudiada, por lo tanto podrían ser aplicadas para la evaluación del IFG en sujetos sanos en la práctica clínica habitual. Si bien la 
ecuación CKD-EPI-CREAT+CIS es la que mostró mejor rendimiento diagnóstico (Tabla 4), la diferencia observada con CKD-EPI-CREAT no es clínicamente relevante, siempre que se cuente con una determinación de creatinina sérica automatizada y trazable con el método de referencia, por lo que debería evaluarse el costo-beneficio de adicionar la medición de Cistatina $\mathrm{C}$ en la estimación de la función renal en la población con FG mayores a $60 \mathrm{ml} / \mathrm{min} / 1,73 \mathrm{~m}^{2}$.

Conclusiones

En una población local con función renal cercana a la normal las ecuaciones CKD-EPI-CREAT, CKD-EPI-CREAT+CIS y STEVENS-CREAT+CIS permiten una estimación del IFG clínicamente aceptable, siempre que se cuente con una determinación de creatinina sérica automatizada y trazable con el método de referencia.

El agregado de la determinación de Cistatina C en la ecuación CKD-EPI combinada mejora la especificidad en el diagnóstico de ERC estadio II pero con un mayor costo.

El presente trabajo aporta al conocimiento del desempeño de las ecuaciones de estimación del IFG en nuestra población.

\section{Los autores declaran no tener conflictos de intereses.}

\section{Bibliografía}

1. Kidney Disease Outcome Quality Initiative (KVDOQI). Clinical practice guidelines for chronic kidney disease: evaluation, classification and stratification. Am J Kidney Dis 2002; 39: S76-S110.

2. Gozdowska J, Urbanowicz A, Sadowska A et al. Glomerular filtration rate estimation in prospective living kidney donors: preliminary study. Transplantation proceedings 2014; 46: 2592-2597.

3. Luján P, Chiurchiu C, Douthat W et al. Ckd-epi instead of mdrd for candidates to kidney donation. Transplantation 2012; 94: 637-641. 4. Sandllands E, Dhaun N, Dear J et al. Measurement of renal function in patients with chronic kidney disease. Br J Clin Pharmacol 2013; 76: 504-515.

5. Dharnidharka V, Kwon $C$ and Stevens G. Serum cystatin $c$ is superior to serum creatinine as a marker of kidney function: a metaanalysis. Am J Kidney Dis 2002; 40: 221-226.

6. Fernández García M, Coll E, Ventura Pedret S et al. Cistatina c en la evaluación de la función renal. Rev Lab Clin 2011; 4: 50-62.

7. Van Rossum L, Zietse R, Vulto A et al. Renal extraction of cystatin c vs 125l-iothalamate in hypertensive patients. Nephrol Dial Transplant 2006; 21: 1253-1256.

8. Alles A, Fraga A, García R et al. Detección precoz de enfermedad renal crónica. Acta Bioquím Clín Latinoam 2010; 44: $377-384$.

9. Levey A, Coresh J, Greene $T$ et al. Expressing the modification of diet in renal disease study equation for estimating glomerular filtration rate with standarized serum creatinine values. Clin Chem 2007; 53: 766-772.

10. Levey A, Stevens L, Schmid C et al. A new equation to estimate glomerular filtration rate. Ann Intern Med 2009; 150: 604-612.

11. Simonsen $\mathrm{O}$, Grubb $A$ and Thysell $\mathrm{H}$. The blood serum concentration of cystatin c (gamma-trace) as a measure of the glomerular filtration rate. Scand J Clin Lab Invest 1985; 45: 97-101.

12. Newman D. Cystatin c. Ann Clin Biochem 2002; 39: 89-104.

13. Bouvet $Y$, Bouissou F, Coulais $Y$ et al. Gfr is better estimated by considering both serum cystatin $c$ and creatinine levels. Pediatr Nephrol 2006; 21: 1299-1306.

14. Sterner G, Björk J, Carlson J et al. Validation of new plasma cystatin c - based formula and the Modification of Diet in Renal Disease creatinine - based formula for determination of glomerular filtration rate. Scand J Urol Nephrol 2009: 43: 242-249.

15. Hoek F, Kemperman $F$ and Krediet $R$. A comparison between cystatin c, plasma creatinine and the cockcroft and gault formula for the estimation of glomerular filtration rate. Nephrol Dial Transplant. 2003; 18: 2024-2031.

16. Herget-Rosenthal S, Bökenkamp A and Hofmann W. How to estímate gfr-serum creatinine, serum cystatin $c$ or equations?. Clin Biochem 2007; 40: 153-161.

17. Filler $G$ and Lepage $N$. Should the schwartz formula for estimation of gfr be replaced by cystatin c formula?. Pediatr Nephrol 2003; 18: $981-985$.

18. Stevens L, Coresh J, Schmid $C$ et al. Estimating gfr using serum cystatin $c$ alone and in combination with serum creatinine: a pooled analysis of 3418 individuals with ckd. Am J Kidney Dis 2008; 51: 395-406.

19. Larsson A, Malm J, Grubb A et al. Calculation of glomerular filtration rate expressed in $\mathrm{ml} / \mathrm{min}$ from plasma cystatin $c$ values in $\mathrm{mg} / \mathrm{L}$. Scand J Clin Invest 2004; 64: 25-30.

20. Inker L, Schmid C, Tighiouart $H$ et al. Estimating glomerular filtration rate from serum creatinine and cystatin c. N Engl J Med 2012; 367: 20-29.

21. Chiurchiu C, Garcés N, Garay G et al. Utilidad de las ecuaciones basadas en la concentración sérica de cistatina c en el estudio de la función renal. Medicina 2007; 67: 136-142.

22. Mahmoud O, Wael A, Gamal A et al. Evaluation of serum cystatin $c$ as a marker of early renal impairment in patients with liver cirrosis. International J Hepatol 2015; 2015: 1-8.

23. Krones $E$, Fickert $P$, Zitta $S$ et al. The chronic kidney disease epidemiology collaboration equation combining creatinine and cystatin $c$ accurately assesses renal function in patients with cirrosis. BioMed Central Nephrol 2015; 16: 196.

24. Momtaz H, Dehghan A and Karimian M. Correlation of cystatin $c$ and creatinine based estimates of renal function in children with hydronephrosis. J Renal Inj Prev 2016; 5:25-28.

25. Satirapoj B, Jirawatsiwaporn K, Tangwonglert $T$ et al. Performance of the estimated glomerular filtration rate creatinine and cystatin $c$ based equations in Thai patients with chronic glomerulonephritis. Int J Nephrol Renovas Disease 2015; 8: 145-150.

26. Bland J, Altman D. Comparing methods of measurement: why plotting difference against standard method is misleading. Lancet 1995; 346: 1085-1087. 